THU0418

NEUROPATHIC-LIKE PAIN IN PERSONS WITH HAND OSTEOARTHRITIS AND ASSOCIATIONS WITH PERIPHERAL AND CENTRAL SENSITIZATION

Pernille Steen Pettersen ${ }^{1}$, Tuhina Neogi ${ }^{2}$, Marthe Gløersen ${ }^{1}$, Karin Magnusson ${ }^{1,3}$, Hilde Berner Hammer ${ }^{1}$, Tore K. Kvien ${ }^{1}$, Till Uhlig ${ }^{1}$, Ida Kristin Haugen'.

${ }^{1}$ Diakonhjemmet Hospital, Rheumatology, Oslo, Norway, ${ }^{2}$ Boston University School of Medicine, Clinical Epidemiology Research and Training Unit, Boston, United States of America; ${ }^{3}$ Lund University, Faculty of Medicine, Clinical Sciences Lund, Orthopaedics, Clinical Epidemiology, Lund, Sweden

Background: Despite no current knowledge of lesions or disease of the sensory nervous system in osteoarthritis $(\mathrm{OA})$, subgroups of knee $O A$ patients report neuropathic-like pain. The neuropathic-like pain component has been suggested to reflect central sensitization.

Objectives: To describe the prevalence of neuropathic-like pain in persons with hand $\mathrm{OA}$ and explore whether neuropathic-like pain associate with peripheral and/or central sensitization.

Methods: These cross-sectional analyses included 280 participants with hand $\mathrm{OA}$ from the Nor-Hand study. A modified PainDETECT questionnaire was used to characterize neuropathic-like hand pain (mPD-hand, 0 36 scale). We conducted quantitative sensory testing (QST) of pressure pain thresholds (PPT) and temporal summation (TS). Low PPTs at local sites (painful finger joint and non-painful finger joint) indicate peripheral and/or central sensitization, while low PPTs at extra-segmental sites (radioulnar joint, musculus trapezius and tibialis anterior) indicate central sensitization. TS, defined as an increase in pain $\geq 2$ on a numeric rating scale (NRS, 0-10) during a repetition of ten punctate stimuli at the wrist, indicate central sensitization. Participants with painful hand OA (NRS of hand pain above the patient acceptable symptom score of $\geq 4$ ), were categorized as having either nociceptive pain (mPD-hand $\leq 12$ ) or neuropathic-like pain (mPD-hand score $>12$ ). We examined whether reporting nociceptive or neuropathic-like pain, as compared to no/mild pain (NRS hand pain below 4) was associated with QST results using regression analyses.

Results: In this study sample, $88 \%$ were female, median age was 61 (IQR 57-66) years and $94 \%$ fulfilled the ACR criteria for hand OA. Median mPD-hand score was 8 (IQR 3, 13). Half of the participants $(n=144,51 \%)$ reported NRS pain $\geq 4$, of whom $58(40 \%)$ had neuropathic-like pain. Those with neuropathic-like pain reported higher mean (SD) NRS pain severity than persons with nociceptive pain $(5.9$ (1.6) vs 5.3 (1.5), $p=0.01$ ). We found that neuropathic-like pain was associated to higher pain sensitization reflected by lower PPT at the painful finger joint (Table), non-painful finger joint (beta $-1.6,95 \% \mathrm{Cl}-2.3,-0.9$ ), radioulnar joint (beta $-1.1,95 \% \mathrm{Cl}-1.8,-0.4$ ), trapezius (Table) and tibialis anterior $(-1.0,95 \% \mathrm{Cl}-1.8,-0.2)$ and presence of TS (Table). The associations between nociceptive pain and sensitization were weaker than the associations observed for neuropathic-like pain (Table).

Abstract THU0418 -Table 1. Associations between neuropathic-like pain and quantitative sensory testing

\begin{tabular}{lcccccc}
\hline & \multicolumn{2}{c}{$\begin{array}{c}\text { PPT painful finger } \\
\text { joint }\end{array}$} & \multicolumn{2}{c}{$\begin{array}{c}\text { PPT trapezius } \\
\text { muscle }^{1}\end{array}$} & $\begin{array}{c}\text { Temporal } \\
\text { summation }\end{array}$ \\
\hline & $\begin{array}{c}\text { Mean } \\
\text { sean }\end{array}$ & $\begin{array}{c}\text { beta(95\% } \\
\text { Cl) }\end{array}$ & $\begin{array}{c}\text { Mean } \\
\text { (SD) }\end{array}$ & $\begin{array}{c}\text { beta(95\% } \\
\text { Cl) }\end{array}$ & $\mathrm{N}(\%)$ & $\begin{array}{c}\text { OR(95\% } \\
\text { Cl) }\end{array}$ \\
\hline No/Mild pain & 4.3 & 0.0 (ref.) & 4.9 & 0.0 (ref.) & 43 & 0.0 (ref.) \\
$(\mathrm{n}=136)$ & $(2.1)$ & & $(2.1)$ & & $(32 \%)$ & \\
Nociceptive pain & 3.9 & $-0.5(-1.0$, & 4.1 & $-0.6(-1.1$, & 35 & $1.3(0.7$ \\
$(\mathrm{n}=86)$ & $(1.7)$ & $0.1)$ & $(1.9)$ & $0.0)$ & $(41 \%)$ & $2.4)$ \\
Neuropathic-like pain & 2.9 & $-1.6(-2.2,-$ & 3.6 & $-0.9(-1.5,-$ & 39 & $3.8(1.8$ \\
$(\mathrm{n}=58)$ & $(1.4)$ & $0.9)$ & $(1.9)$ & $0.2)$ & $(67 \%)$ & $8.2)$ \\
\hline
\end{tabular}

${ }^{1}$ Linear regression, ${ }^{2}$ logistic regression. Adjusted for age, sex, BMI, use of analgesics, level of education, sleep disturbance, Pain Catastrophizing Scale, the Hospital Anxiety and Depression Scale and radiographic hand OA severity (KellgrenLawrence sum score).

Conclusion: Of persons with painful hand OA, $40 \%$ have a neuropathiclike pain component. Reporting neuropathic-like pain is associated with peripheral and central sensitization, indicated by low PPTs and presence of TS. Our results suggest that patients who report neuropathic-like symptoms are more likely to have sensitization-associated pain.

Disclosure of Interests: Pernille Steen Pettersen: None declared, Tuhina Neogi: None declared, Marthe Gløersen: None declared, Karin Magnusson: None declared, Hilde Berner Hammer Grant/research support from: AbbVie, Pfizer and Roche, Paid instructor for: AbbVie, Pfizer, UCB, Novartis, Roche, Speakers bureau: AbbVie, Pfizer, UCB, Novartis, Roche, Tore K. Kvien Grant/research support from: AbbVie, BMS, MSD, Pfizer, Roche and UCB., Consultant for: AbbVie, Biogen, BMS, Boehringer
Ingelheim, Celgene, Celltrion, Eli Lilly, Hospira, Merck-Serono, MSD, Novartis, Oktal, Orion Pharma, Pfizer, Roche, Sandoz, Sanofi, Mylan and UCB, Speakers bureau: AbbVie, Biogen, BMS, Boehringer Ingelheim, Celgene, Celltrion, Eli Lilly, Hospira, Merck-Serono, MSD, Novartis, Oktal, Orion Pharma, Pfizer, Roche, Sandoz, Sanofi and UCB, Till Unlig Consultant for: Grünenthal, Novartis, Speakers bureau: Grünenthal, Novartis, Ida Kristin Haugen Grant/research support from: ADVANCE research grant from Pfizer, Consultant for: Advisory board Abbvie

DOI: 10.1136/annrheumdis-2019-eular.4060

\section{THU0419 BIOMARKERS OF BONE AND CARTILAGE TURNOVER CTX-I AND CTX-II PREDICT TOTAL JOINT REPLACEMENTS IN OA}

Jonathan Bjerre-Bastos ${ }^{1}$, Anne-Christine Bay-Jensen ${ }^{2}$, Morten Karsdal ${ }^{2}$, Inger Byrjalsen ${ }^{1}$, Jeppe Ragnar Andersen ${ }^{1}$, Bente Juel Riis ${ }^{3}$, Claus Christiansen ${ }^{3}$, Asger Reinstrup Bihlet ${ }^{1}{ }^{1}$ Nordic Bioscience, Clinical Development, Herlev, Denmark, ${ }^{2}$ Nordic Bioscience, Biomarkers and Research, Herlev, Denmark; ${ }^{3}$ Nordic Bioscience, Herlev, Denmark

Background: Osteoarthritis $(\mathrm{OA})$ can lead to joint failure and ultimately total joint replacement (TJR). Identifying risk-factors of developing joint failure is of interest for clinicians and researchers. Currently late-stage clinical trial design for evaluating new treatments in $O A$ is evolving with the possible utilization of accelerated approval pathways. This may require outcome studies with longer duration, in which joint failures may qualify as an endpoint. However, TJRs are relatively rare events and are known to be biased by doctor/patient interactions as well as local practice guidelines, and consequently, there is a need for objective non-invasive measures, such as soluble biomarkers (BM), to enrich the population for this outcome. BM may act as important tools in investigating the association between biochemical/pathological processes and risk of joint failure. Degradation of collagens type I and II are known to be involved in OA disease progression, and the biomarkers CTX-I (C-telopeptide of crosslinked collagen type I), a marker of bone degradation, and CTX-II (C-telopeptide of crosslinked collagen type II), a marker of cartilage degradation, may reflect disease progression with clinical relevance for the risk of joint failure.

Objectives: To evaluate baseline (BL) BM SCTX-I and UCTX-II as predictors of TJR.

Methods: Data from two clinical trials investigating oral salmon calcitonin (SMC) in OA, NCT00486434 and NCT00704847, was analyzed post-hoc. Data was dichotomized by the bottom and top quartile of the respective $\mathrm{BM}$ concentration at BL to compare the number TJRs of the knee or hip in groups with high vs. low SCTX-I or UCTX-II, respectively. For means of visualization, plotted in Kaplan-Meier curves.

Cox Proportional Hazard Regression was performed to determine the association of BL SCTX-I and UCTX-II to the incidence of TJR while adjusting for co-variates; age, BMI, and gender. Data from both treatment groups were analyzed. Only the first reported incident was included in the analysis for each subject.

Results: A total of 68 TJRs of knee or hip were reported, of which 49 were knees and 18 were hips. One patient underwent two TJRs, resulting in 67 subjects with events.

Results from the Cox Proportional Hazard Regression adjusted for covariates of age, BMI, and sex (table 1), indicate that high BL SCTX-I compared to low was associated with a 3.4 times higher risk of undergoing an arthroplasty of the knee or hip within a two-year period $(p=0.04)$. High BL UCTX-II compared to low was associated with a 3.1 times higher risk of undergoing a TJR of the knee or hip during the study period $(p=0.04)$, and an 8.9-fold increased risk of undergoing a knee replacement during the study period $(\mathrm{p}=0.02)$.

TJR events over time in groups of high or low UCTX-II through the study are illustrated in figure 1.

Abstract THU0419 -Table 1. Cox Proportional Hazards Regression analysis

\begin{tabular}{|c|c|c|c|c|c|c|}
\hline \multirow[t]{2}{*}{ Group } & \multicolumn{3}{|c|}{ Low CTX-I $\begin{aligned} N & =336, \text { high CTX-I } \\
N & =340\end{aligned}$} & \multicolumn{3}{|c|}{ Low CTX-II $\begin{aligned} N & =318, \text { high CTX-\| } \\
N & =322\end{aligned}$} \\
\hline & $\begin{array}{c}\text { HR top vs. } \\
\text { bottom quartile }\end{array}$ & $\begin{array}{c}95 \% \\
\mathrm{Cl}\end{array}$ & $\begin{array}{c}\mathrm{P}- \\
\text { value }\end{array}$ & $\begin{array}{c}\text { HR top vs. } \\
\text { bottom quartile }\end{array}$ & $\begin{array}{c}95 \% \\
\mathrm{Cl}\end{array}$ & $\begin{array}{c}P_{-} \\
\text {value }\end{array}$ \\
\hline $\begin{array}{l}\text { Knee or hip } \\
\text { replacement } \\
\text { (Cases: CTX-I = 23, } \\
\text { CTX-II = 27) }\end{array}$ & 3.40 & $\begin{array}{l}1.05- \\
11.06\end{array}$ & 0.042 & 3.08 & $\begin{array}{l}1.04- \\
9.10\end{array}$ & 0.043 \\
\hline $\begin{array}{l}\text { Knee replacement } \\
\text { (Cases: CTX-I = 16, } \\
\text { CTX-II = 19) }\end{array}$ & 2.72 & $\begin{array}{l}0.70- \\
10.57\end{array}$ & 0.15 & 8.94 & $\begin{array}{l}1.50- \\
53.48\end{array}$ & 0.017 \\
\hline
\end{tabular}


Total joint replacements

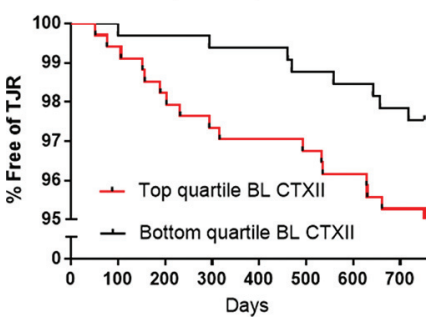

Abstract THU0419 - Figure 1. TJR events during the entire study, CTX-II.

Conclusion: High levels of SCTX-I and UCTX-II at BL were associated with increased risk of undergoing TJR of the knee or hip during the two important biomarkers in clinical OA trials evaluating incidence of TJRs.

Disclosure of Interests: : Jonathan Bjerre-Bastos Employee of: I am a full-time employee in Nordic Bioscience, Anne-Christine Bay-Jensen Shareholder of: I own shares of Nordic Bioscience, Employee of: I am a fulltime employee in Nordic Bioscience, Morten Karsdal Shareholder of: I own shares of Nordic Bioscience, Employee of: I am a full-time employee in Nordic Bioscience, Inger Byrjalsen Employee of: I am a full-time employee in Nordic Bioscience, Jeppe Ragnar Andersen Shareholder of: Nordic Bioscience, Employee of: Nordic Bioscience, Bente Juel Riis Shareholder of: Yes, I own shares in Nordic Bioscience, Employee of: I am a full-time employee in Nordic Bioscience, Claus Christiansen Shareholder of: Yes, I own shares in Nordic Bioscience, Employee of: I am a full-time employee in Nordic Bioscience, Asger Reinstrup Bihlet Shareholder of: Yes, I own shares in Nordic Bioscience., Employee of: I am a full-time employee in Nordic Bioscience

DOI: 10.1136/annrheumdis-2019-eular.7306

\section{THU0420 METABOLIC SYNDROME AND TRAJECTORIES OF LOCALISED PAIN AND GENERALISED PAIN}

Feng Pan ${ }^{1}$, Jing Tian ${ }^{1}$, Flavia Cicuttini ${ }^{2}$, Graeme Jones ${ }^{1} .{ }^{1}$ University of Tasmania, Medical School, Department of Epidemiology and Preventive Medicine, Melbourne, Australia

Background: Metabolic syndrome (MetS) has been suggested as having a link to the pathophysiology of pain; however, no study has assessed generalised pain and their courses over time.

Objectives: To describe the associations of MetS and its components with trajectories of localised knee pain (pain severity) and generalised pain ((number of painful sites (NPS)) in a general older population.

Methods: 1,099 participants from a population-based older adult cohort study were recruited at baseline. 875, 768 and 563 participants attended years 2.6, 5.1 and 10.7 follow-up, respectively. Data were collected on demographic, psychological, lifestyle and comorbidities, blood pressure, glucose, triglycerides, and high-density lipoprotein (HDL) cholesterol. MetS was defined based on the National Cholesterol Education Program-Adult Treatment Panel III criteria. Radiographic knee osteoarthritis (ROA) was assessed by X-ray. Knee pain was measured by Western Ontario and McMaster Universities Osteoarthritis Index pain questionnaire at each time-point. Presence/absence of pain at the neck, back, hands, shoulders, hips, knees and feet was collected by questionnaire at each time-point. year study. Our findings support the role of SCTX-I and UCTX-II as Menzies Institute for Medical Research, Hobart, Australia; ${ }^{2}$ Monash University whether MetS and its components are associated with localised pain and

Group-based trajectory modelling was applied to identify pain trajectories. Multi-nominal logistic regression was used for the analyses.

Results: Of 985 participants included in this study, 32\% of participants had MetS and $60 \%$ had ROA at baseline. Three localised knee pain severity trajectories were identified: 'Minimal pain' $(52 \%)$, 'Mild pain' $(33 \%)$ and 'Moderate pain' (15\%). Three NPS trajectories were identified: 'Low NPS' (12\%), 'Medium NPS' (38\%), and 'High NPS' (49\%). In multivariable analysis without adjusting for central obesity, central obesity increased risk of belonging to both 'Mild pain' and 'Moderate pain' trajectories as compared to the 'Minimal pain' trajectory group, but MetS, hypertriglyceridemia and low HDL were only associated with 'Moderate pain' trajectory [relative risk $(R R): 1.67-2.26$, all $P<0.05$ ]. Similarly, central obesity was also associated with both 'Medium NPS' (RR 2.35, 95\% Cl 1.40-3.92) and 'High NPS' trajectories (RR $3.07,95 \% \mathrm{Cl} 1.85-5.08$ ) compared to 'Low NPS' trajectory group, whereas MetS was only associated with 'High NPS' trajectory (RR 2.60, 95\% Cl 1.54-4.41). These associations became weak and non-significant after further adjustment for central obesity.

Conclusion: MetS is predominantly associated with trajectories of localised and generalised pain through central obesity, suggesting that weight management is important in the prevention and therapy of pain over time.

Disclosure of Interests: None declared

DOI: 10.1136/annrheumdis-2019-eular.1245

\section{THU0421 EFFECT OF GAME BASED EXERCISE PROGRAMS ON PAIN, FUNCTIONAL MOBILITY AND BALANCE IN PATIENTS WITH KNEE OSTEOARTHRITIS: RANDOMIZED CONTROLLED STUDY}

DEMIRiz SERAP YILMAZ1', Ali Erdem Baki'. ' Zonguldak Atatürk Public Hospital, Physical Medicine and Rehabilitation, Zonguldak, Turkey; ${ }^{2}$ Zonguldak Bulent Ecevit University Faculty of Medicine, Physical Medicine and Rehabilitation, Zonguldak, Turkey

Background: Osteoarthritis is a chronic joint disease affecting the knee, hip and hand joints. Exercise is an integral component of conservative treatment for osteoarthritis. However, virtual reality applications using interactive games for rehabilitation have become a focus of interest in recent years.

Objectives: The aim of this study was to evaluate the effects of virtual reality games on knee pain, functional mobility and balance in patients with knee osteoarthritis.

Methods: Fifty patients who were complaining of knee pain, aged 40-70 years, and were diagnosed with Kellgren-Lawrence stage 2, 3, 4 idiopathic knee osteoarthritis was included. Patients were randomly assigned to two equal groups $(n=25)$ as the control and the study group. Age, sex, weight, height, duration of illness was recorded for all patients. In the bilateral two-way knee radiography; Kellgren-Lawrence score of the knee with the higher visual analog scale (VAS) pain score was recorded. Conventional physical therapy program, associated with knee osteoarthritis exercises, was applied routinely to all patients with knee osteoarthritis for three weeks. In the study group, all patients received virtual reality game-based exercise program by using a game console. In both groups, baseline and final values of VAS score, osteoarthritis index total score (WOMAC), and intra-community balance and mobility scale (CB\&M) score of each patient were recorded. Mann-Whitney $U$ and Independent Samples $T$ tests were used for statistical analysis, and $p$ value less than 0.05 was considered significant.

Results: In both groups, significant change in VAS, WOMAC osteoarthritis index, and CB\&M score was observed $(p<0.05)$. In the study group, VAS, WOMAC osteoarthritis index, and CB\&M scores were significantly different than control group $(p<0.05)$. The differences between baseline

Abstract THU0429 - Table 1. Results of four separate multiple linear regression models with the WOMAC as the dependent variable. Regression coefficients with 95\% confidence intervals are shown.

\begin{tabular}{|c|c|c|c|c|c|c|}
\hline & \multirow[b]{2}{*}{ Parameter } & \multirow[b]{2}{*}{ Reference } & \multicolumn{4}{|c|}{ WOMAC } \\
\hline & & & Polyarthrosis & Hip OA & Knee and Hip OA & Knee OA \\
\hline \multirow[t]{2}{*}{ Model 1} & Age & per 10 years & $0,7(-0,6 ; 2,0)$ & $0,7(-1,2 ; 2,6)$ & $2,0(-0,5 ; 4,4)$ & $-0,1(-3,5 ; 3,3)$ \\
\hline & Gender: Male & Female & $-3,8(-6,7 ;-1,0)$ & $0,8(-3,5 ; 5,0)$ & $-3,7(-8,1 ; 0,6)$ & $-0,5(-7,6 ; 6,6)$ \\
\hline Model $2^{*}$ & $\mathrm{BMI}, \mathrm{kg} / \mathrm{m}^{2}$ & per 5 units & $4,4(3,2 ; 5,5)$ & $3,1(1,1 ; 5,2)$ & $4,2(1,8 ; 6,6)$ & $4,1(0,6 ; 7,6)$ \\
\hline Model $3^{*}$ & Symptom duration & per year & $0,3(0,2 ; 0,5)$ & $0,4(0,2 ; 0,6)$ & $0,2(-0,0 ; 0,4)$ & $0,4(0,0 ; 0,8)$ \\
\hline Model $4^{*}$ & WHO-5 (0-100) & per $10 \%$ worsening & $4.4(3.8 ; 5.0)$ & $3.2(2.2 ; 4.1)$ & $4.1(3.3 ; 5.1)$ & $3.9(2.1 ; 5.9)$ \\
\hline
\end{tabular}

*Model adjusted for age, gender. BMI: Body Mass Index, OA: Osteoarthritis, WOMAC: Western Ontario and McMaster Universities Osteoarthritis Score. 